\title{
Using social media to assess the consumer nutrition environment: comparing Yelp reviews with a direct observation audit instrument for grocery stores
}

\author{
Ying Shen ${ }^{1}$, Philippa Clarke ${ }^{1,2, *}$, Iris N Gomez-Lopez ${ }^{2}$, Alex B Hill ${ }^{3}$, Daniel M Romero ${ }^{4}$, \\ Robert Goodspeed ${ }^{5}$, Veronica J Berrocal ${ }^{6}$, VG Vinod Vydiswaran ${ }^{4,7}$ \\ and Tiffany $C$ Veinot $^{4,8}$ \\ ${ }^{1}$ Department of Epidemiology, University of Michigan, Ann Arbor, MI, USA: ${ }^{2}$ Institute for Social Research, University \\ of Michigan, 426 Thompson Street, Ann Arbor, Ml 48104, USA: ${ }^{3}$ Detroit Food Map Initiative, Detroit, MI, USA: \\ ${ }^{4}$ School of Information, University of Michigan, Ann Arbor, MI, USA: ${ }^{5}$ Taubman College of Architecture and Urban \\ Planning, University of Michigan, Ann Arbor, MI, USA: ${ }^{6}$ Department of Biostatistics, University of Michigan, Ann \\ Arbor, MI, USA: ${ }^{7}$ Department of Learning Health Sciences, University of Michigan, Ann Arbor, MI, USA: \\ ${ }^{8}$ Department of Health Behavior and Health Education, University of Michigan, Ann Arbor, MI, USA
}

Submitted 11 February 2018: Final revision received 21 September 2018: Accepted 25 September 2018: First published online 8 November 2018

\begin{abstract}
Objective: To examine the feasibility of using social media to assess the consumer nutrition environment by comparing sentiment expressed in Yelp reviews with information obtained from a direct observation audit instrument for grocery stores. Design: Trained raters used the Nutrition Environment Measures Survey in Stores (NEMS-S) in 100 grocery stores from July 2015 to March 2016. Yelp reviews were available for sixty-nine of these stores and were retrieved in February 2017 using the Yelp Application Program Interface. A sentiment analysis was conducted to quantify the perceptions of the consumer nutrition environment in the review text. Pearson correlation coefficients $(\rho)$ were used to compare NEMS-S scores with Yelp review text on food availability, quality, price and shopping experience.

Setting: Detroit, Michigan, USA.

Participants: None.

Results: Yelp reviews contained more comments about food availability and the overall shopping experience than food price and food quality. Negative sentiment about food prices in Yelp review text and the number of dollar signs on Yelp were positively correlated with observed food prices in stores $(\rho=0.413$ and 0.462 , respectively). Stores with greater food availability were rated as more expensive on Yelp. Other aspects of the food store environment (e.g. overall quality and shopping experience) were captured only in Yelp.

Conclusions: While Yelp cannot replace in-person audits for collecting detailed information on the availability, quality and cost of specific food items, Yelp holds promise as a cost-effective means to gather information on the overall cost, quality and experience of food stores, which may be relevant for nutrition outcomes.
\end{abstract}

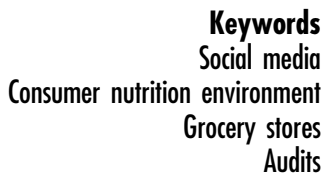

A large body of research indicates that the nutrition environment, including the availability of food stores and restaurants within a community, and the quality and price of healthy food choices within these establishments, influences eating behaviour ${ }^{(1-7)}$. Food stores that sell foods and beverages that can be prepared at home are an important element of the nutrition environment, particularly in the light of evidence that suggests at-home food preparation is associated with better dietary intake and more family meals ${ }^{(8,9)}$.
Measures of the nutrition environment are necessary for understanding the factors influencing healthy eating behaviour $^{(10)}$. Researchers have used geographic-based measures to capture access to community food sources, including distance to the nearest supermarket, density of food stores within a given area and gravity-based measures that also incorporate travel time and modality ${ }^{(11-18)}$. The availability, price and quality of healthy options within food stores represent the consumer nutrition environment, which reflects what consumers actually encounter within a 
retail food store ${ }^{(10)}$. However, measurement of the consumer nutrition environment can be challenging because of the large number of potential factors that are believed to be related to the purchase of healthy foods (e.g. price, promotions, placement of items within a store, range of choices, freshness, visibility of nutritional information $)^{(10,19)}$. In-person audits have therefore been considered the gold standard to fully document what consumers can actually purchase inside a store ${ }^{(20,21)}$.

Direct observational audits, such as the widely used Nutrition Environment Measures Survey in Stores (NEMSS) ${ }^{(19,22)}$, provide objective and rigorous assessments of the consumer nutrition environment ${ }^{(23)}$. Trained raters use the NEMS-S to rate the price and availability of ten food categories and assess the quality of fresh fruits and vegetables in food stores ${ }^{(24)}$. The NEMS-S is one of the few audit tools with demonstrated reliability and validity and has been used repeatedly in research on the consumer nutrition environment ${ }^{(22,24)}$. However, onsite assessments with in-person audits are time-intensive and costly, especially when auditing a large number of stores across a wide geographic area ${ }^{(22)}$.

The vast amount of web-based information on social media holds promise as a cost-effective alternative to inperson audits of the consumer nutrition environment. Social media, such as Twitter, Facebook and Yelp, are web-based forms of communication where people share information and create content ${ }^{(25)}$. A growing body of research has used geographically referenced social media to assess multiple aspects of the nutrition environment, including food-borne illness outbreaks ${ }^{(26,27)}$, the relationship between healthy food-related Twitter posts and proximity to healthy food stores ${ }^{(28)}$, and to create neighbourhood indicators of healthy food availability ${ }^{(29,30)}$. Researchers have documented associations between the prevalence of healthy food-related postings on social media and the socio-economic characteristics of the local neighbourhood ${ }^{(29,31,32)}$. Recent work has also shown a relationship between food-related social media posts and county- and state-level health outcomes ${ }^{(25,33)}$.

However, no research to date has explored the use of social media for assessing the nutritional content and offerings inside a community food store. The purpose of the present study was to examine the feasibility of using Yelp to assess the consumer nutrition environment. Yelp (www.yelp.com) is a popular social media site that provides a platform for consumers to post reviews of local businesses and services. In 2017, Yelp averaged over 75 million unique users per month ${ }^{(34)}$. User-generated content includes an overall business rating (1 to 5 stars), cost rating ( 1 to 4 dollar signs, representing 'inexpensive', 'moderate', 'pricey' and 'ultra high-end', respectively) and detailed text reviews from users that capture cost, quality and other aspects of the business experience.

We used Yelp reviews to assess the consumer nutrition environment for sixty-nine grocery stores in the City of
Detroit, Michigan, USA. Detroit is a rich focus for this work since it has experienced dramatic structural and economic decline since the 1950s, with consequences for the availability and quality of healthy food sources for local residents ${ }^{(35)}$. Using sentiment analysis ${ }^{(36)}$ we mined the Yelp review text for indicators of the consumer nutrition environment, including healthy food availability, price and quality. We then assessed the degree to which Yelp review metrics were consistent with NEMS-S scores obtained from a direct observation audit of these sixty-nine stores.

\section{Methods}

We focused on 'full-line' grocery stores, following the Michigan Department of Agriculture \& Rural Development's definition as 'a store selling fresh produce, fresh meat, fresh bread, and fresh dairy' ${ }^{\text {(37) }}$. An enumerative list of 102 full-line grocery stores in Detroit, Michigan was obtained from Detroit Food Map (http://www.detroit foodmap.com/), a community-based initiative that assesses the quality of food stores as access points for nutritious and healthy food options in Metropolitan Detroit. Trained raters conducted NEMS-S audits at 102 grocery stores from July 2015 to March 2016. Audits were not completed for two stores, leaving 100 stores with complete NEMS-S audits.

A total of sixty-nine of the 100 grocery stores with complete audit data had records on Yelp. The Yelp Application Program Interface (Yelp Fusion API; https:// www.yelp.com/fusion) was used in February 2017 to request the online public information for each of these sixty-nine grocery stores. Metadata for each store (including name, address, number of reviews and store ratings) were retrieved using the Yelp API. Yelp review text was retrieved by separately downloading and parsing the review pages for each store.

\section{NEMS-S measures}

The NEMS-S scoring system considers the availability, quality and price of healthy choices within ten food categories: milk, fruits, vegetables, ground beef, hot dogs, baked goods, beverages, bread, potato chips and cer$\mathrm{eal}^{(19,38)}$. Separate scores for availability, price and quality are created and summed to create an overall total score ${ }^{(19)}$. Higher scores indicate greater availability and quality and lower cost of healthy options.

NEMS-S availability scores are based on the number of different varieties of fruits and vegetables, as well as the presence of healthier options within each of the nonproduce food categories (i.e. low-fat/skimmed milk, lean ground beef, fat-free hot dogs, low-fat baked goods, $100 \%$ juice or diet soda, wholegrain bread, baked chips and lowsugar cereal). The total availability score ranges from 0 to 
27 with a higher score indicating a greater availability of healthier options.

NEMS-S quality scores are assigned for fruits and vegetables based on the proportion of produce that is rated either acceptable or unacceptable (score $=3$ if $>75 \%$ acceptable; score $=2$ if $50-75 \%$ acceptable; score $=1$ if $<50 \%$ acceptable). A total quality score is calculated by summing the scores for fruits and vegetables (range 0-6).

NEMS-S price scores are based on the relative price of the healthier option within each non-produce food category (e.g. skimmed milk $v$. whole milk). The scoring system assigns negative values if the cost of healthier options is greater than the cost of comparable regular options ${ }^{(19)}$.

Total NEMS-S scores were calculated by summing the availability, price and quality scores for five key food categories (milk, fruits, vegetables, ground beef and bread). A maximum score of 39 reflects greater availability of, and relatively cheaper prices for, more healthful or recommended food choices and quality produce. Because not all stores had healthier options for sale in all five food categories, total NEMS-S scores were available for only fifty-six of the sixty-nine stores.

Food prices (in US dollars) were calculated for the healthy items in each of six food categories most relevant for nutrition (low-fat milk, fruits, vegetables, lean ground beef, wholegrain bread and cereal). Because not all items were available in all stores, only forty-five stores had data on healthy food prices. An overall total healthy food price for each store was created by generating $Z$-scores to standardize the prices across the different food categories. The total food price was calculated by summing the $Z$-scores of each food category for each store.

\section{Yelp measures}

For each store, summary Yelp metrics were calculated for overall store rating (number of stars out of 5), cost rating (number of dollar signs out of 4) and total number of reviews. A sentiment analysis was then conducted with the Yelp review text to capture the perceptions of the consumer nutrition environment with respect to the dimensions of the NEMS-S (food availability, price, quality). Sentiment related to the overall shopping experience was also included to tap other dimensions of the consumer nutrition environment not assessed by the NEMS-S (e.g. customer service, cleanliness, spaciousness, crowdedness) that could be important for food store choices ${ }^{(39)}$.

Sentiment analysis refers to the use of natural language processing techniques to systematically identify and quantify information and opinions from web-based textual information $^{(36,40-42)}$. We used a food-related subset of the Linguistic Inquiry and Word Count (LIWC) tool ${ }^{(43)}$ to generate a comprehensive list of 594 keywords, phrases and adjacency expressions capturing both positive and negative sentiment about (i) food availability, (ii) price, (iii) quality and (iv) shopping experience. We first eliminated LIWC keywords that did not correspond to the NEMS-S categories (e.g. words describing food preparation: 'braised', 'scalded'). We then manually examined the review phrases containing frequently used keywords $(>10$ occurrences) to confirm that they were being used to describe concepts in the NEMS-S. If needed, keywords were expanded to better capture sentiment, such as by adding phrases and adjacency expressions (e.g. 'not very fresh' $v$. 'fresh'). Table 1 shows examples of positive and negative sentiment keywords in each of the four dimensions, along with illustrative examples from the Yelp review text.

For each dimension, we calculated a positive sentiment score and a negative sentiment score based on the proportion of positive and negative keywords, respectively, in each store's reviews. To adjust for the number of reviews per store and the length of each review, we normalized the sentiment scores using the Okapi BM25 method (a text retrieval algorithm), which scales the total number of keywords in a store's review by the total number of words in the review relative to the average length of the review across all sixty-nine stores ${ }^{(44,45)}$. Weighted proportions were then averaged across all reviews in each store to capture the average positive and negative sentiment on food availability, price, quality and general shopping experience. Net sentiment was also computed by subtracting the average negative sentiment score from the average positive sentiment score for each dimension. All sentiment scores are expressed in percentages.

\section{Statistical analyses}

Descriptive statistics (means and standard deviations) were used to summarize the consumer nutrition environment according to the NEMS-S scores and the Yelp review metrics. Pearson correlation coefficients $(\rho)$ were used to assess the agreement of the sentiment scores from the Yelp reviews with the NEMS-S availability scores, total NEMS-S scores and food price $Z$-scores. All analyses were conducted using the statistical software package SAS version 9.4. Statistical significance of the correlation coefficients was assessed with an $\alpha$ level of $0 \cdot 05$.

\section{Results}

Summary statistics for the consumer nutrition environment in these sixty-nine grocery stores are presented in Table 2. NEMS-S availability scores ranged from 8 to 17 , with a mean score of 12.9 out of a possible score of 27 , reflecting a lack of availability of a large range of healthier options (Table 2). This low availability was also reflected in the total NEMS-S scores for five key food categories in the NEMS-S (milk, fruits, vegetables, ground beef, bread), 
Table 1 Illustrative Yelp review text and sentiment keywords for food availability, price, quality and shopping experience from the sample of sixty-nine full-line Detroit area grocery stores, Michigan, USA, February 2017

Food availability

Examples of positive sentiment text in Yelp review

Examples of positive sentiment keywords ( $n$ 69)

Examples of negative sentiment text in Yelp review

Examples of negative sentiment keywords ( $n$ 38)

Food prices

Examples of positive sentiment text in Yelp review

Examples of positive sentiment keywords ( $n$ 45)

Examples of negative sentiment text in Yelp review

Examples of negative sentiment keywords ( $n$ 20)

Food quality

Examples of positive sentiment text in Yelp review

Examples of positive sentiment keywords ( $n$ 32)

Examples of negative sentiment text in Yelp review

Examples of negative sentiment keywords ( $n$ 89)

Shopping experience

Examples of positive sentiment text in Yelp review

Examples of positive sentiment keywords ( $n 55)$

Examples of negative sentiment text in Yelp review

Examples of negative sentiment keywords ( $n$ 39)
'They offer a HUGE variety of fresh meat, seafood, cheese and breads.'

'Beautiful, bountiful produce galore (literally saw the biggest tomatoes I've ever seen in my life). 'Located in the heart of Arab America, this grocery store offers an excellent selection of fruits, vegetables, meats, and dairy.'

'large assortment', 'array', 'fresh produce', 'low-fat dairy', 'lean meats', 'whole grain', 'nutritious', 'variety', 'healthy', 'heart smart', 'good selection'

'I do find that this particular Meijer is often out of products, which can be annoying and result in me having to make a trip to another store.'

'It's still disappointing how many holes there are in their inventory (basics like heavy whipping cream, and brands like Morningstar Farms meat substitutes), and indeed, it's a rare meal where I can find all the ingredients needed here.'

'They do exist, but the selection isn't vast like it is in most "American" grocery stores.'

'can't find', 'not much', 'oily', 'salty', 'don't have', 'low on stock', 'junky', 'carries fewer', 'tiny selection'

'Amazing prices for very good produce.'

'Food Pride is super big too, it really has everything you are looking for AND it's super cheap, you know mama loveeeees her a good deal.'

'The prices of the meat, on sale, are excellent.'

'bargain', 'cheap', 'deals', 'good deal', 'great value', 'reasonable', 'save', 'specials', 'savings', 'save'

'Meat is expensive, no breaks there either.'

'You can find any grocery item that you need there, though it may be the ridiculously overpriced organic version that you don't really want.'

'I totally understand why prices might be higher for a city-based store but some items are ridiculously overpriced.'

'expensive', 'costly', 'higher end', 'hit to the wallet', 'isn't worth', 'rip off', 'overpriced'

'I was so impressed with the prices, selection, and quality of the fresh produce.'

'Their produce also all looked delicious and was well priced.'

I love Super Greenland for the fresh produce and freshly ground meat along with all the other delicious goodies that can be had here.'

'fresh', 'good produce', 'quality', 'nice looking', 'tender'

'Additionally, there have been numerous times when I have seen fresh produce covered in mould or simply past ripe.'

'Warning: I bought some of the dried Turkish figs and found a couple of them had little spider nests inside and another was very sour almost like it was fermenting or just rotten.'

'... every time I get home, I look at the packaging and the food is expired.'

'bruised', 'curdled', 'damaged', 'expired', 'fatty', 'greasy', 'mould', 'not fresh', 'old', 'rotten', 'smelly', 'wilted'

'What rocks: Decent, fresh food, reasonably priced, presented in a convenient and attractive setting WITHIN the city of Detroit.'

'I've always had great service here - employees stop \& offer help and they always seem happy to do so.'

'It's very clean, the layout makes sense and it's pretty easy to find things.'

'amazing customer service', 'bright', 'clean', 'friendly', 'nearby', 'spacious', 'not crowded'

'The back area is filthy, the bottle room stinks like rotten garbage and the bathroom was a disgusting mess.'

'Now the stock for everything else is reduced significantly, the aisles are tiny (half the time I have to leave my cart since I can't manoeuvre it around in the cramped space), and it's just such a bummer to shop at.'

'The store is dirty, staff is rude and groceries are very expensive.'

'crowded', 'grungy', 'impolite', 'messy', 'dirty', 'rude', 'slow', 'unclean', 'wait'
$Z$-score US dollars (mean $=0.5, \mathrm{sD}=8 \cdot 0)$. Results of unstandardized price comparisons (not shown in Table 2) indicated that healthier food options tended to be more expensive than less healthy options. Over two-thirds of the stores sold whole-wheat bread, lean ground beef, produce quality. On average, total NEMS-S scores were

$18 \cdot 3$ out of a possible score of 39.

Food prices across six food categories (milk, fruits, vegetables, ground beef, bread, cereal) were captured in 
$100 \%$ juice and low-fat chips at higher prices than their less-healthy counterparts.

The mean number of Yelp reviews per store was $20 \cdot 6$ (range 1-172) with half the stores having fewer than five

Table 2 Summary statistics of NEMS-S scores and Yelp review metrics for the sample of sixty-nine full-line Detroit area grocery stores, Michigan, USA, July 2015-March 2016 and February 2017

\begin{tabular}{lrr}
\hline & Mean & SD \\
\hline NEMS-S scores & & \\
NEMS-S availability score & 12.9 & 3.31 \\
Total NEMS-S score & 18.3 & 2.63 \\
Food prices (Z-score US dollars) & 0.5 & 8.03 \\
Yelp review metrics & & \\
Number of reviews & 20.6 & 32.50 \\
Number of stars (out of 5) & 3.3 & 0.96 \\
Number of dollar signs (out of 4) & 1.6 & 0.58 \\
Yelp review sentiment & & \\
Food availability sentiment (\%) & & \\
Positive & 34.4 & 0.19 \\
Negative & 7.6 & 0.12 \\
Net & 26.8 & 0.19 \\
Food price sentiment (\%) & & \\
Positive & 19.9 & 0.19 \\
Negative & 3.4 & 0.06 \\
Net & 16.5 & 0.19 \\
Food quality sentiment (\%) & & \\
Positive & 14.8 & 0.16 \\
Negative & 6.6 & 0.12 \\
$\quad$ Net & 8.2 & 0.19 \\
Shopping experience sentiment (\%) & & \\
Positive & 25.9 & 0.18 \\
Negative & 8.4 & 0.12 \\
Net & 17.5 & 0.19 \\
\hline
\end{tabular}

NEMS-S, Nutrition Environment Measures Survey in Stores. reviews (Table 2). The mean store rating on Yelp was 3.3 stars out of a possible 5 stars. Mean store cost, reflected through the number of dollar signs in the review, was 1.6, representing 'inexpensive' to 'moderate' prices according to Yelp.

Table 2 presents the average percentage of positive and negative sentiment words (normalized) in the Yelp review text across each of the four dimensions (availability, price, quality, overall shopping experience). In general, reviews contained more sentiment words about food availability and the overall shopping experience than about food price and food quality. In addition, positive sentiment in the reviews was greater than negative sentiment for each dimension. For example, the average proportion of positive sentiment words for food availability was $34.4 \%$ in the Yelp reviews compared with $7 \cdot 6 \%$ for negative sentiment. The net availability sentiment (26.8\%) reflects the dominance of positive words related to food availability over negative words. Similarly, for the overall shopping experience, almost $26 \%$ of the review text contained positive words and $8.4 \%$ contained negative words, for a net general sentiment of $17.5 \%$ on average.

Table 3 presents the Pearson correlation coefficients for the NEMS-S scores and the Yelp review metrics. (Stratified analyses were also conducted by the number of Yelp reviews per store ( $\leq 5 v .>5$ reviews), but there was no difference in the pattern of results. Therefore, only the unstratified results are presented in Table 3.) Stores with higher availability scores on the NEMS-S tended to be rated as more expensive on Yelp (i.e. more dollar signs; $\rho=0.444$, Table 3). Similarly, higher total NEMS-S scores

Table 3 Pearson correlation coefficients for Yelp metrics and NEMS-S scores for the sample of sixty-nine full-line Detroit area grocery stores, Michigan, USA, July 2015-March 2016 and February 2017

\begin{tabular}{|c|c|c|c|}
\hline & \multicolumn{3}{|c|}{ NEMS-S scores } \\
\hline & NEMS-S availability score & Total NEMS-S score & Food prices (Z-score US dollars) \\
\hline \multicolumn{4}{|l|}{ Yelp review metrics } \\
\hline Number of reviews & 0.170 & $0.447^{\star \star \star}$ & $0.454^{\star \star}$ \\
\hline Number of stars (out of 5 ) & -0.1143 & 0.049 & $0.317^{\star}$ \\
\hline Number of dollar signs (out of 4 ) & $0.444^{\star \star *}$ & $0.501^{\star \star \star}$ & $0.462^{\star *}$ \\
\hline \multicolumn{4}{|l|}{ Yelp review text sentiment } \\
\hline \multicolumn{4}{|l|}{ Food availability sentiment } \\
\hline Positive & -0.035 & -0.062 & 0.089 \\
\hline Negative & -0.189 & -0.190 & 0.053 \\
\hline Net & 0.081 & 0.073 & 0.058 \\
\hline \multicolumn{4}{|l|}{ Food price sentiment } \\
\hline Positive & -0.092 & -0.117 & -0.089 \\
\hline Negative & 0.108 & 0.262 & $0.413^{\star \star}$ \\
\hline Net & $-0 \cdot 120$ & -0.194 & -0.208 \\
\hline \multicolumn{4}{|l|}{ Food quality sentiment } \\
\hline Positive & $-0.239^{*}$ & -0.235 & -0.028 \\
\hline Negative & -0.103 & -0.101 & -0.152 \\
\hline Net & $-0 \cdot 137$ & -0.127 & 0.062 \\
\hline \multicolumn{4}{|l|}{ Shopping experience sentiment } \\
\hline Positive & 0.017 & 0.053 & $-0 \cdot 136$ \\
\hline Negative & $0.253^{*}$ & 0.116 & -0.039 \\
\hline Net & -0.146 & -0.026 & -0.100 \\
\hline
\end{tabular}

NEMS-S, Nutrition Environment Measures Survey in Stores.

Correlation was statistically significant: ${ }^{\star} P<0.05,{ }^{\star \star} P<0.01,{ }^{\star \star \star} P<0.001$. 
$(\rho=0.501)$, which incorporate produce quality and relative food price in addition to availability, also tended to be rated as more expensive on Yelp. These positive correlations suggest that stores selling a greater variety of healthier food choices with high-quality produce tended to be rated as more expensive. The number of Yelp dollar signs was also positively correlated with the actual food prices in the stores determined through direct observation $(\rho=0.462)$ and negative sentiment about food prices in Yelp review text was positively correlated with higher food prices $(\rho=0.413$, Table 3$)$.

Higher store food prices ( $Z$-score US dollars) were positively associated with higher store ratings (i.e. number of stars; $\rho=0.317$ ) and a greater number of reviews posted for that store $(\rho=0.454$, Table 3). Thus, more expensive stores tended to be those that were rated highly and also those for which people posted more reviews on Yelp. This is consistent with the positive association between the total NEMS-S scores and the number of reviews ( $\rho=0.447$, Table 3), suggesting that people post more reviews for stores that have higher-quality and more healthy foods, which also tend to be pricier.

A greater availability of healthier food choices (reflected in higher NEMS-S availability scores) was negatively associated with positive review sentiment on food quality $(\rho=-0.239)$ and positively associated with more negative sentiment on the shopping experience $(\rho=0.253$, Table 3 ). This suggests that the availability score in the NEMS-S (which is driven by all ten categories of foods, including chips, soda and hot dogs) may not reflect food quality in the eyes of the consumer and stores selling these foods may not provide a quality shopping experience. There were no statistically significant correlations between the food quality review sentiment and total NEMS-S scores (which factor in produce quality only, Table 3 ).

\section{Discussion}

The present study is one of the first to assess the feasibility of using social media to assess the consumer nutrition environment. While social media is increasingly used to assess the nutrition environment with respect to foodborne illness outbreaks ${ }^{(26)}$ and state-level health outcomes $^{(25)}$, no research has explored the potential of using social media to assess the nutritional content and offerings inside food stores. Using sentiment analysis, a method of computational linguistics and text analysis, we compared Yelp review text with NEMS-S scores collected through inperson audits on the availability, price and quality of healthy food options in sixty-nine grocery stores in Detroit, Michigan, USA.

We found that grocery stores that were rated as more expensive on Yelp tended to have higher observed food prices documented during the in-person audit. A larger number of dollar signs on a store's Yelp page was positively correlated with higher observed food prices for fruits, vegetables, milk, beef and bread. Similarly, more negative sentiment expressed about food prices in the Yelp review text was associated with higher food prices observed in the in-person audit. Thus, Yelp review text and overall cost ratings show promise as a reasonable barometer of the cost of healthy food options actually observed in local grocery stores.

We found no correlation between NEMS-S availability scores and Yelp review text sentiment about food availability. Nor did we find any correlation between total NEMS-S scores and Yelp review text sentiment on food price, food quality or food availability (all of which factor into the total NEMS-S score). Thus, Yelp reviews do not appear to be capturing the availability and quality of healthy food choices within stores, as reflected in the NEMS-S scoring system. However, we did find that stores reviewed as more costly on Yelp (reflected through negative price sentiment and a greater number of dollar signs) were more likely to have higher NEMS-S availability scores and higher total NEMS-S scores. This is consistent with other research demonstrating the cost burden of purchasing healthy food ${ }^{(46)}$ and suggests that grocery stores with a greater availability of quality, healthy food choices also tended to be rated on social media as more expensive overall.

We also showed that Yelp reviews can be used to capture additional metrics about the consumer nutrition environment that are not included in the NEMS-S, including general store appearance, cleanliness and service, which have been shown to be important for consumer food store choices ${ }^{(39,47)}$. While the NEMS-S assesses the quality of fruits and vegetables only, Yelp reviews contained information about the quality of other food categories (e.g. the quality of meat $)^{(39,48)}$ and on the overall shopping experience.

Yelp reviews contained more positive sentiment than negative sentiment, suggesting that more positive reviews are posted about grocery stores than negative reviews. Stores with more Yelp reviews and higher store ratings (as reflected by the number of stars on Yelp) also tended to have more expensive food prices observed in the store. Thus, online users may be more inclined to review more high-quality and expensive grocery stores. Alternatively, if these higher quality stores are located in more socioeconomically affluent neighbourhoods, then a greater number of reviews could reflect the tendency of residents in these neighbourhoods to post more reviews on social media. In supplemental analyses (not shown) we found that grocery stores located in more affluent neighbourhoods (captured through census tract indicators of higher education, higher household income and more residents with professional occupations) had significantly more Yelp reviews. Conversely, those stores located in more socio-economically disadvantaged neighbourhoods (captured through census tract indicators of poverty, 
unemployment and more residents on public assistance income) had significantly fewer reviews, consistent with the concept of the 'digital divide'(49) . Differences in both computer literacy and Internet access across these types of neighbourhoods may drive the number of online reviews about the consumer nutrition environment ${ }^{(50,51)}$.

\section{Limitations}

Yelp reviews were not available for all 100 grocery stores in our sample. The thirty-one stores without reviews were significantly more likely to be in socio-economically disadvantaged census tracts and in areas with low broadband adoption $^{(52)}$, suggesting that social media may be useful for assessing the consumer nutrition environment only in less disadvantaged areas. Further research in communities other than Detroit is needed to determine the extent to which social media is selectively used across communities with different characteristics. Although our study was exploratory, we conducted a large number of statistical tests. Thus, the statistical significance of the observed correlations may reflect a statistical anomaly.

\section{Conclusion}

Despite these limitations, the present study is the first attempt to consider whether information gleaned from social media could be useful for evaluating the consumer nutrition environment. Researchers today can draw on an expanding set of data about the nutrition environment, including social media websites like Yelp, which rely on user contributions ${ }^{(30)}$. The study suggests that, while Yelp cannot replace in-person audits for collecting detailed information on the availability, quality and cost of specific food items, Yelp does hold promise as a cost-effective means to gather information on the overall cost and quality of the consumer nutrition environment. Simple metrics like the number of dollar signs on Yelp or the overall star rating may be useful for researchers or practitioners who are unable to conduct in-person audits in community food stores due to time or cost reasons. Even for those able to conduct detailed observational audits, Yelp may serve to supplement NEMS-S metrics of the consumer nutrition environment by providing other indicators of store and food quality relevant for consumer nutrition.

\section{Acknowledgements}

Financial support: This work was funded in part by the Gordon and Betty Moore Foundation through a grant to the University of Michigan (grant number GBMF3943); the Alfred P. Sloan Foundation (grant number 2014-5-05 DS); MCubed and the University of Michigan Office of Research; and the University of Michigan Rackham
Graduate School via the Social Sciences Annual Institute Competition Round 6. The funders had no role in the design, analysis or writing of this article. Conflict of interest: None. Authorship: Y.S., P.C., I.N.G.-L., V.G.V.V. and T.C.V. contributed to the conception and design of the study, data acquisition, and data analysis and interpretation; the writing and revision of the article for important intellectual content; and approved the final version of the submitted manuscript. A.B.H. contribution to data acquisition, the revision of the article for important intellectual content, and read and approved the final version of the submitted manuscript. D.M.R., R.G. and V.J.B. contributed to the interpretation of the data and analysis, revised the article for important intellectual content, and read and approved the final version of the submitted manuscript. Ethics of buman subject participation: Not applicable.

\section{References}

1. Burns C, Bentley R, Thornton L et al. (2015) Associations between the purchase of healthy and fast foods and restrictions to food access: a cross-sectional study in Melbourne, Australia. Public Health Nutr 18, 143-150.

2. Caldwell EM, Kobayashi MM, DuBow W et al. (2009) Perceived access to fruits and vegetables associated with increased consumption. Public Health Nutr 12, 1743-1750.

3. Morland K, Roux AVD \& Wing S (2006) Supermarkets, other food stores, and obesity: the atherosclerosis risk in communities study. Am J Prev Med 30, 333-339.

4. Morland K, Wing S \& Diez Roux AV (2002) The contextual effect of the local food environment on residents' diets. Am J Public Health 82, 1761-1767.

5. Booth SL, Sallis JF, Ritenbaugh C et al. (2001) Environmental and societal factors affect food choice and physical activity: rationale, influences, and leverage points. Nutr Rev 59, $3 \mathrm{Pt}$ 2, S21-S39.

6. Liese AD, Bell BA, Barnes TL et al. (2014) Environmental influences on fruit and vegetable intake: results from a path analytic model. Public Health Nutr 17, 2595-2604.

7. Morland KB, Filomena S, Granieri E et al. (2017) Environmental disparities in the objectively and longitudinally measured local food environments of urban older adults. Med Res Arch 5, issue 7, https://www.journals.ke-i.org/ index.php/mra/article/download/1363/1120/

8. Berge JM, MacLehose RF, Larson N et al. (2016) Family food preparation and its effects on adolescent dietary quality and eating patterns. $J$ Adolesc Health 59, 530-536.

9. Monsivais P, Aggarwal A \& Drewnowski A (2014) Time spent on home food preparation and indicators of healthy eating. Am J Prev Med 47, 796-802.

10. Glanz K, Sallis JF, Saelens BE et al. (2005) Healthy nutrition environments: concepts and measures. Am J Health Promot 19, 330-333.

11. Barnes TL, Bell BA, Freedman DA et al. (2015) Do people really know what food retailers exist in their neighborhood? Examining GIS-based and perceived presence of retail food outlets in an eight-county region of South Carolina. Spat Spatiotemporal Epidemiol 13, 31-40.

12. Barnes TL, Freedman DA, Bell BA et al. (2016) Geographic measures of retail food outlets and perceived availability of healthy foods in neighbourhoods. Public Health Nutr 19, 1368-1374.

13. Van Meter E, Lawson AB, Colabianchi N et al. (2011) Spatial accessibility and availability measures and statistical 
properties in the food environment. Spat Spatiotemporal Epidemiol 2, 35-47.

14. Wilkins EL, Morris MA, Radley D et al. (2017) Using Geographic Information Systems to measure retail food environments: discussion of methodological considerations and a proposed reporting checklist (Geo-FERN). Health Place 44, 110-117.

15. Chen X (2017) Take the edge off: a hybrid geographic food access measure. Appl Geogr 87, 149-159.

16. Ma X, Liese AD, Bell BA et al. (2016) Perceived and geographic food access and food security status among households with children. Public Health Nutr 19, 2781-2788.

17. McKinnon RA, Reedy J, Morrissette MA et al. (2009) Measures of the food environment: a compilation of the literature, 1990-2007. Am J Prev Med 36, 4 Suppl., S124-S133.

18. Lytle LA \& Sokol RL (2017) Measures of the food environment: a systematic review of the field, 2007-2015. Health Place 44, 18-34.

19. Glanz K, Sallis JF, Saelens BE et al. (2007) Nutrition Environment Measures Survey in stores (NEMS-S): development and evaluation. Am J Prev Med 32, 282-289.

20. Moudon AV, Drewnowski A, Duncan GE et al. (2013) Characterizing the food environment: pitfalls and future directions. Public Health Nutr 16, 1238-1243.

21. Gustafson AA, Sharkey J, Samuel-Hodge CD et al. (2011) Perceived and objective measures of the food store environment and the association with weight and diet among low-income women in North Carolina. Public Health Nutr 14, 1032-1038.

22. Partington SN, Menzies TJ, Colburn TA et al. (2015) Reduced-item food audits based on the Nutrition Environment Measures Surveys. Am J Prev Med 49, e23-e33.

23. Ohri-Vachaspati P \& Leviton LC (2010) Measuring food environments: a guide to available instruments. Am J Health Promot 24, 410-426.

24. Honeycutt S, Davis E, Clawson M et al. (2010) Training for and dissemination of the Nutrition Environment Measures Surveys (NEMS). Prev Chronic Dis 7, A126.

25. Nguyen QC, Meng H, Li D et al. (2017) Social media indicators of the food environment and state health outcomes. Public Health 148, 120-128.

26. Nsoesie EO, Kluberg SA \& Brownstein JS (2014) Online reports of foodborne illness capture foods implicated in official foodborne outbreak reports. Prev Med 67, 264-269.

27. Harrison C, Jorder M, Stern H et al. (2014) Using online reviews by restaurant patrons to identify unreported cases of foodborne illness - New York City, 2012-2013. MMWR Morb Mortal Wkly Rep 63, 441-445.

28. Chen X \& Yang X (2014) Does food environment influence food choices? A geographical analysis through 'tweets'. Appl Geogr 51, 82-89.

29. Nguyen QC, Kath S, Meng H-W et al. (2016) Leveraging geotagged Twitter data to examine neighborhood happiness, diet, and physical activity. Appl Geogr 73, 77-88.

30. Gomez-Lopez IN, Clarke P, Hill AB et al. (2017) Using social media to identify sources of healthy food in urban neighborhoods. J Urban Health 94, 429-436.

31. Widener MJ \& Li W (2014) Using geolocated Twitter data to monitor the prevalence of healthy and unhealthy food references across the US. Appl Geogr 54, 189-197.

32. Ghosh D \& Guha R (2013) What are we 'tweeting' about obesity? Mapping tweets with topic modeling and Geographic Information System. Cartogr Geogr Inf Sci 40, 90-102.

33. Nguyen QC, McCullough M, Meng H-W et al. (2017) Geotagged US tweets as predictors of county-level health outcomes, 2015-2016. Am J Public Health 107, 1776-1782.
34. Yelp.com (2017) An introduction to Yelp Metrics as of September 30, 2017. https://www.yelp.com/factsheet (accessed February 2018).

35. Zenk SN, Schulz AJ, Israel BA et al. (2005) Neighborhood racial composition, neighborhood poverty, and the spatial accessibility of supermarkets in metropolitan Detroit. $\mathrm{Am} \mathrm{J}$ Public Health 95, 660-667.

36. Cambria E, Schuller B, Xia Y et al. (2013) New avenues in opinion mining and sentiment analysis. IEEE Intell Syst $\mathbf{2 8}$, $15-21$.

37. Michigan Department of Agriculture (2012) Michigan's food \& agriculture industry. http://www.michigan.gov/documents/ mdard/1262-AgReport-2012_2_404589_7 (accessed March 2016).

38. Glanz K, Basil M, Maibach E et al. (1998) Why Americans eat what they do: taste, nutrition, cost, convenience, and weight control concerns as influences on food consumption. J Am Diet Assoc 98, 1118-1126.

39. Krukowski RA, Sparks C, DiCarlo M et al. (2013) There's more to food store choice than proximity: a questionnaire development study. BMC Public Health 13, 586.

40. Jensen PB, Jensen LJ \& Brunak S (2012) Mining electronic health records: towards better research applications and clinical care. Nat Rev Genet 13, 395-405.

41. Carrell DS, Cronkite D, Palmer RE et al. (2015) Using natural language processing to identify problem usage of prescription opioids. Int J Med Inform 84, 1057-1064.

42. Grafton JR, Yu O, Carrell D et al. (2016) Identifying race/ ethnicity data via natural language processing among women in a uterine fibroid cohort study. J Patient Cent Res Rev 3, 226.

43. Pennebaker JW, Chung CK, Ireland $\mathrm{M}$ et al. (n.d.) The Development and Psychometric Properties of Liwc 2007. http://hdl.handle.net/2152/31333 (accessed March 2016).

44. Jones KS, Walker S \& Robertson SE (2000) A probabilistic model of information retrieval: development and comparative experiments - Part 1. Inf Process Manage 36, 779-808.

45. Robertson SE (1990) On term selection for query expansion. J DOC 46, 359-364.

46. Rao M, Afshin A, Singh G et al. (2013) Do healthier foods and diet patterns cost more than less healthy options? A systematic review and meta-analysis. BMJ Open 3, e004277.

47. Young T, Swanson A, Craven T et al. (2014) Food habits of residents who live near corner stores (132.3). FASEB J $\mathbf{2 8}$, 132.133.

48. Krukowski RA, West DS, Harvey-Berino J et al. (2010) Neighborhood impact on healthy food availability and pricing in food stores. J Community Health 35, 315-320.

49. Chang BL, Bakken S, Brown SS et al. (2004) Bridging the digital divide: reaching vulnerable populations. J Am Med Inform Assoc 11, 448-457.

50. Jensen JD, King AJ, Davis LA et al. (2010) Utilization of internet technology by low-income adults: the role of health literacy, health numeracy, and computer assistance. J Aging Health 22, 804-826.

51. Henly S, Tuli G, Kluberg SA et al. (2017) Disparities in digital reporting of illness: a demographic and socioeconomic assessment. Prev Med 101, 18-22.

52. Veinot TC, Goodspeed R, Clarke P et al. (2017) Information and Education Environment. Neighborbood Effects: Community Characteristics and Health in Metropolitan Detroit. Ann Arbor, MI: University of Michigan Libraries; available at https://deepblue.lib.umich.edu/data/concern/generic_works/ $3 \mathrm{f} 462547 \mathrm{~h}$ 